\title{
A case of alar stenosis: single-staged alar reconstruction
}

\author{
Ji-Woong Cho, $\mathrm{MD}^{1} \mathbb{1}$, Hye Jeen Kim, $\mathrm{MD}^{1} \mathbb{1}$, Do-Yoon Jeong, $\mathrm{MD}^{1} \mathbb{1}$, Ibrahim AlQuniabut, $\mathrm{MD}^{2} \mathbb{1}$, \\ Ji Yun Choi, MD, PhD' ${ }^{1}$ (10 \\ 'Department of Otolaryngology-Head and Neck Surgery, Chosun University College of Medicine, Gwangju, Rep. of Korea \\ ${ }^{2}$ Department of Surgery, Unaizah College of Medicine and Medical Sciences, Qassim University, Buraydah, Kingdom of Saudi Arabia
}

\begin{abstract}
The ala is a cosmetically and functionally critical part of the nose, with unique esthetic and anatomical characteristics. Herein, we report our experience of alar reconstruction. A 56-year-old female was diagnosed with intranasal mucosal melanoma and was treated by surgical excision and radiotherapy. She developed left nostril deformity after the completion of oncological treatment. She underwent single-staged alar reconstruction surgery using alar rotational and turning-in flaps with septal and conchal cartilage reconstruction of the ala and lower lateral cartilage. A defect of the ala as a result of cancer excision or trauma is a challenging entity in facial reconstruction. In addition, radiotherapy is a burden for major reconstructive flaps because of defective healing. Hence, singlestaged local flaps with septal and conchal cartilage reconstruction results in good outcome and fewer complications.
\end{abstract}

Keywords: external nasal valve; nasal reconstruction; nostril; nostril reconstruction; rhinoplasty; stenosis of nostril

\section{Introduction}

The ala is an important landmark of the nose and face both functionally and cosmetically. Functionally, the external nasal valve is framed by ala. Cosmetically, the important visual landmarks are the transition from supra-alar shadow to convex reflection and the distal free edge of the alar lobule.

Cancer of the nose can cause functional and cosmetic concerns after excision, particularly defects of the alar subunit. Preoperative recognition of whether reconstruction of the defect requires skin coverage, cartilage support, internal lining, or a combination of these allows optimum results.

The altered landmarks of the ala not only disturb the esthetics of the face, but also the function of the nose. As a result, nasal reconstruction is more complicated compared toother parts of the face, as the skin coverage, bone-cartilage framework, and mucosal lining of the nose must be restored accordingly $[1,2]$.
Reconstructive options include various flaps or graft repairs, depending on the subunit involvement, defect size, patient comorbidity, and surgeon experience. While primary closures may be suitable for small defects, more complex single or twostage flaps may be considered for moderate to large defects involving partial and full-thickness defects of the ala.

\section{Case report}

A 56-year-old female visited our facial plastic clinic seeking to correct the left nostril deformity for both esthetic and functional purposes. In 2018, the patient was diagnosed with intranasal mucosal melanoma and underwent surgical resection with selective neck dissection and postoperative radiotherapy. Currently, the patient is under regular surveillance for melanoma.

Physical examination of her ala showed scar tissue over the left alar skin, and the superior vestibular surface was present,

Received November 25, 2021; Revised December 23, 2021; Accepted December 23, 2021

Corresponding author: Ji Yun Choi

E-mail: happyent@naver.com

This is an Open Access article distributed under the terms of the Creative Commons Attribution Non-Commercial License (http://creativecommons.org/licenses/by-nc/4.0), which permits unrestricted non-commercial use, distribution, and reproduction in any medium, provided the original work is properly cited.

Copyright ( 2021 Korean Society of Korean Cosmetic Surgery and Medicine (KSKCS \& KCCS). 
with the alar rim retracted superiorly and posteriorly due to the contracture effect. The nostril was stenotic and narrow with soft triangle blunting, which pulled the tip of the nose and columella to the left side. Abnormal curvature of the alar wall was observed, indicating a misshaped lower lateral cartilage (LLC) (Fig. 1).

The two-dimensional new stenotic nostril was designed based on the contralateral normal nostril. The vertical dimension and width of the nostril opening and soft triangle location were measured (Fig. 2A). The alar rotation flap was designed by utilizing the supra-alar groove to hide the scar (Fig. 2B).

Open-approach rhinoplasty was then performed with a rim incision on the left side and a marginal incision on the right side. Septal cartilage was harvested, and septoplasty was per- formed. Wide undermining of the nasal dorsum and side wall was performed to allow skin mobilization from the lateral nasal side wall and medial cheek.

Intraoperatively, the lateral-most part of the LLC was missing, and the medial part was weak and contracted along the middle crus by scar tissue extending cephalically up to the scroll area with weakness of the caudal margin of the upper lateral cartilage. All scar tissues were removed along with the vestibular tissue.

Re-enforcement of the lateral-most part of the LLC by the harvested septal cartilage and left dome was reconstructed using harvested conchal cartilage as a gently convex curved graft (Fig. 3A). An alar batten graft was used to support the scroll area and prevent future collapse of scar tissue. Finally, tip augmenta-
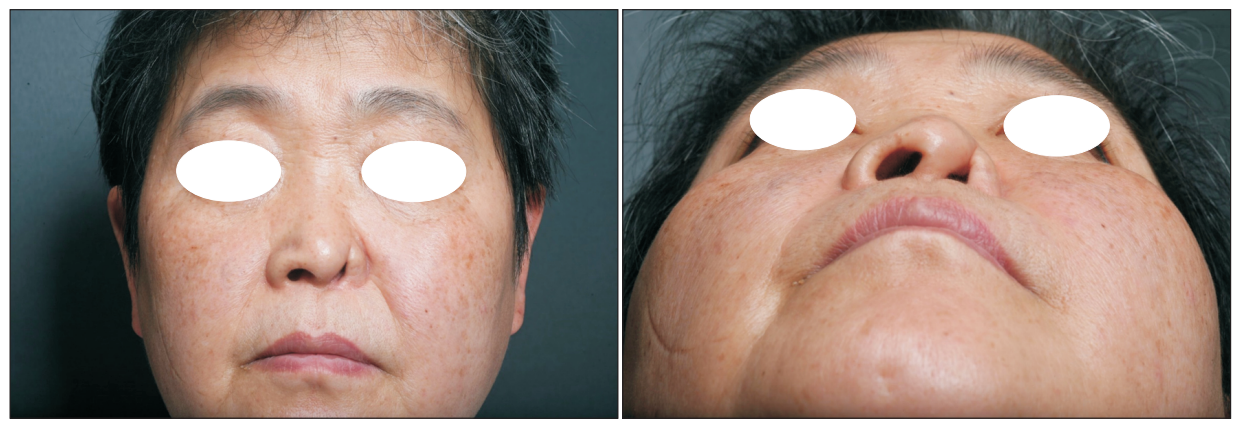

Fig. 1. Preoperative photo. Physical examination of the ala shows scar tissue over the left alar skin and superior vestibular surface, with the alar rim retracted superiorly and posteriorly due to the contracture effect. The nostril is stenotic and narrow with soft triangle blunting, pulling the tip of the nose and columella to the left side. An abnormal curvature of the alar wall is observed, indicating a misshaped lower lateral cartilage.
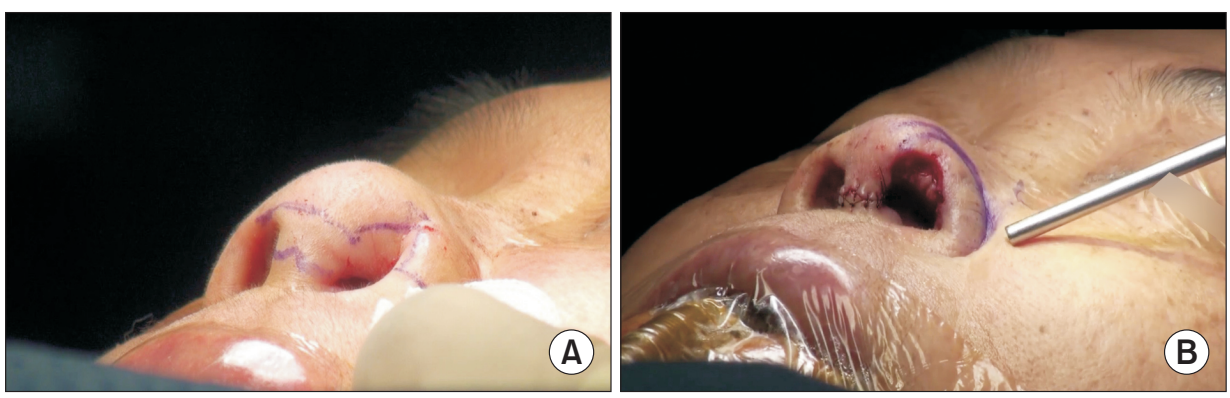

Fig. 2. Incision design. (A) Incision mark for open rhinoplasty and the turnin flap design. The vertical dimension width of the nostril opening and soft triangle location were measured based on the contralateral normal nostrils. (B) Planned design of the alar rotation flap.
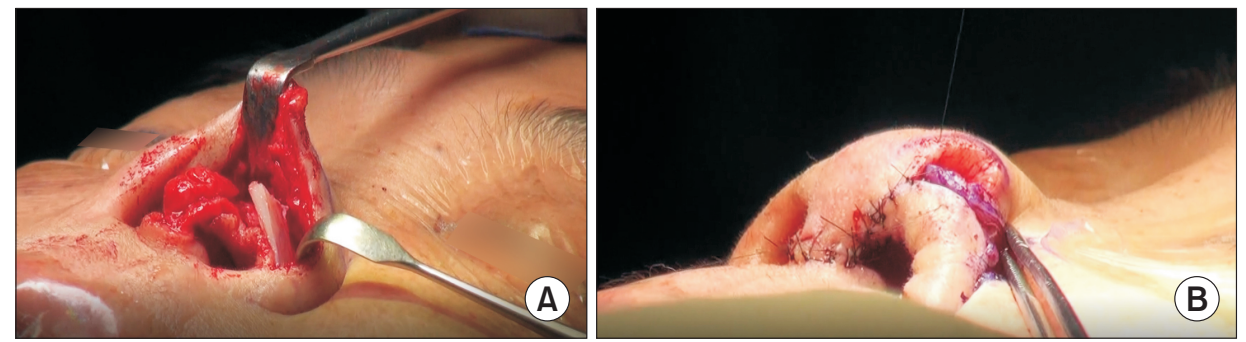

Fig. 3. Reconstruction of lower cartilage using conchal cartilage and alar groove covered by Z-plasty. (A) Re-enforcement graft for the lateralmost part of the lower lateral cartilage by harvested septal cartilage, and the left dome has been reconstructed using harvested conchal cartilage as a gently convex curved graft. The alar rotation flap has been inset to cover the alar rim defect, and Z-plasty has been elevated to cover the secondary defect (B). 
tion and asymmetry were corrected using a tip-only graft.

Reconstruction of the internal lining of the nostril was performed using a skin turn-in flap by advancing the skin from the rim incision and turning the skin of the nostril toward the nasal vestibule (Fig. 3B). Then, alar rotation flap was elevated with laterally based pivot point to cover the donor site (alar rim skin) (Fig. 4A). Nose cheek Z-plasty from the medial cheek to repair the supra-alar groove defect was designed and rotated after wide undermining (Fig. 4B). Finally, the wound was closed with double layer closure, and bolster sutures were used to hold the reconstruction and prevent hematoma at two points (Fig. 5).

During 2nd month of follow-up, the wound healed well and the flap was viable. The function was cosmetically restored with satisfactory outcomes for both the patient and the surgeon (Fig. $6)$.

\section{Discussion}

Successful reconstruction of the ala of the nose requires a comprehensive understanding of both the function and esthetic anatomy. The ala is surrounded by a crescent-like groove and alar-facial sulcus, which serve as topographic landmarks and distinguish it from surrounding esthetic subunits [3]. The ala joins the tip of the nose anterior-medially and the sidewall of the nose superiorly. Moreover, the alar-facial sulcus separates it from the cheek and the upper lip [3].

Nostril deformity is a cosmetically and functionally devastating condition for the patient [4]. The reconstruction of the nostril requires consideration of the mucosal lining, skeletal framework, and skin [5]. Depending on the involved part, defect size, patient comorbidity, and surgeon experience, various procedures exist, but only the use of appropriate procedure and delicate manipulation can yield good results [5]. However, the
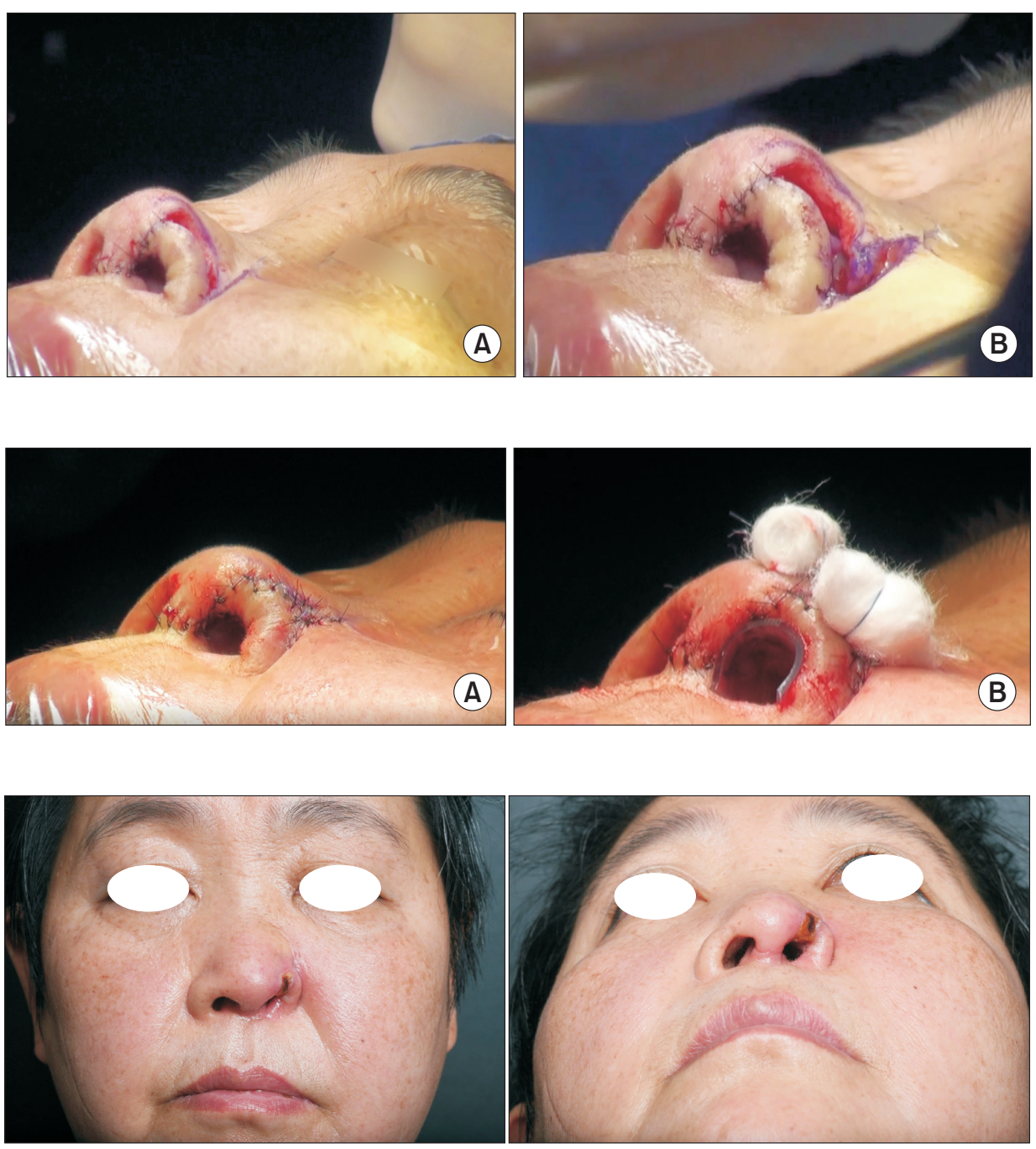

Fig. 4. Nose-cheek Z-plasty. (A) Rotation and inset of the alar rotation flap in the alar rim defect. The Z-plasty has been designed between the junction of the nose-cheek groove, and the flap has been elevated and undermined to allow easy mobility (B).

Fig. 5. Closure of wound and flaps with bolster suture application. (A) The primary defect has been covered by an alar rotation flap, with a secondary alar groove defect corrected by Z-plasty from the medial cheek. (B) A bolster suture has been used to hold the reconstruction and prevent hematoma at two points.

Fig. 6. Postoperative photo. Two months post-surgery, the wound healed well, and the flap was viable. The function was restored cosmetically with a satisfactory outcome. The tip is more defined and centrally located, with a wide and open left nostril. No major scars were visible on patient's face. 
more complaint soft tissue of the ala compared to the tip and sidewall of the nose, as it does not have fixed cartilage, results in relatively variable options for reconstruction.

Anatomically, the lobule of the ala is composed of fat and skeletal muscles enveloped by the dermis and epithelium in both the external and vestibular parts. Furthermore, the alar groove is not crossed by the LLC; hence, it is not part of it. The absence of osseous-cartilaginous structures and the supportfree distal zone makes it very delicate and vulnerable to distortion and very challenging to reconstruct [3]. Nevertheless, the alar lobule gets its stability and support from ligaments and soft tissue attachments to more stable surrounding structures [3].

The ala has a rich blood supply network from both internal and carotid artery systems. The facial and infraorbital arteries make up the majority of the blood supply. Thus, the transpositional and rotational flaps are safe and favorable due to its viability and scar healing capacity [3]. Furthermore, the border of alar lobule is a free margin, which makes the incorporation of alar skin into vestibular skin valuable to recruit adjacent skin without further donor morbidity [6].

In case of partial-thickness alar defects, if the size is less than $1 \mathrm{~cm}$, either primary closure or a conchal cartilage composite graft produces good to excellent results with no complications. For defects larger than $1 \mathrm{~cm}$ but not more than $2 \mathrm{~cm}$, bilobed and nasolabial flaps are the flaps of choice. In case of fullthickness defects, a nasolabial flap was ideal to cover defects of $2 \mathrm{~cm}$ or less, but the forehead flap was the best reconstructive option for defects larger than $2 \mathrm{~cm}$ [7]. A bilobed flap is a variant of the rotational flap, a flap in which two flaps with a common base are migrating. A primary flap is used to fill the defect, and a secondary flap is used to repair the first flap.

Even if the nostril defect is small, the nostrils may bend after recovery if they are not reconstructed in three layers. Full-thickness alar reconstructions are subjected to significant contractile forces during healing. Without proper consideration and structural support, this may lead to significant alar retraction, alar notching, columellar show, and poor cosmetic outcome. The difficulty arises with full-thickness defects because of the need for reconstruction of the internal nasal lining, which, if underestimated, most likely leads to total reconstructive failure [5]. External nasal valve collapse may also accompany reconstruction of the nasal ala, especially when soft tissue flaps are large or poorly supported. To prevent, the use of alar batten grafts, they may be carved from the septal, conchal, or rib cartilage.
The contracture effect of scarring makes skin laxity unfavorable for recruiting it from the same esthetic unit of the nose. Thus, regional flaps have been used to overcome this challenge. Nevertheless, these flaps may need a second stage or result in an unpleasant color or texture mismatch or obliteration of the esthetic subunit border [6]. However, a local flap may be considered to reconstruct nostrils, such as an alar rotation flap. In case of cartilage defects, it is necessary to reconstruct the LLC using septal and/or ear cartilage.

\section{Conclusion}

The ala of the nose is considered a challenging part for reconstruction because of its special anatomical features. Nonetheless, various surgical options and flaps exist in order to repair the defect. However, each option should be carefully selected based on the individual patient to avoid unwanted outcome and complications. In the present case, a local flap with singlestage surgery was preferred because of a history of radiotherapy to the nose, which may lead to future failure of the pedicled flap.

\section{Conflicts of interest}

The authors have nothing to disclose.

\section{References}

1. Burget GC. Aesthetic reconstruction of the tip of the nose. Dermatol Surg 1995;21:419-29.

2. Sherris DA, Larrabee WF Jr. Principles of facial reconstruction: a subunit approach to cutaneous repair. 2nd ed. New York, NY: Thieme; 2009. p. 102-60.

3. Bloom JD, Ransom ER, Miller CJ. Reconstruction of alar defects. Facial Plast Surg Clin North Am 2011;19:63-83.

4. Daines SM, Hamilton GS 3rd, Mobley SR. A graded approach to repairing the stenotic nasal vestibule. Arch Facial Plast Surg 2010;12:332-8.

5. Kim MS, Choi JY. Surgical reconstruction of nasal alar deformities. J Rhinol 2017;24:65-73.

6. Neltner SA, Papa CA, Ramsey ML, Marks VJ. Alar rotation flap for small defects of the ala. Dermatol Surg 2000;26:543-6.

7. Yellin SA, Nugent A. Melolabial flaps for nasal reconstruction. Facial Plast Surg Clin North Am 2011;19:123-39. 